\title{
Synthesis and magnetic properties of stable cobalt nanoparticles decorated reduced graphene oxide sheets in the aqueous medium
}

Neelam Singh ${ }^{1}$, J.R. Ansari ${ }^{1}$, Mrinal Pal ${ }^{2}$, Nguyen TK Thanh ${ }^{3}$, Tung Le ${ }^{4}$, and Anindya Datta ${ }^{1, *}$

${ }^{1}$ University School Basic \& Applied Sciences, Guru Gobind Singh Indraprastha University Dwarka, New Delhi-110078, India

${ }^{2}$ CSIR-Central Glass \& Ceramic Research Institute, Council of Scientific \& Industrial Research, Kolkata-700032, India

${ }^{3}$ UCL Healthcare Biomagnetic and Nanomaterials Laboratories; 3rd Floor, The Royal Institution of Great Britain, 21 Albemarle Street, London W1S 4BS, UK

${ }^{4}$ Dept of Physics \& Astronomy; University College London, Gower Street, London WC1E 6BT, UK

*Corresponding Author: Dr. Anindya Datta, +91-9953408629

Email: anindya_datta@yahoo.com 


\begin{abstract}
We have synthesized cobalt nanoparticles-reduced graphene oxide (Co-RGO) nanocomposites. The Co NPs achieve shape variation in different nanocomposites due to the strategic use of variety in the preparation techniques. The transmission electron microscope image of composites, confirms the decoration of different shapes of Co NPs on RGO sheets. The magnetic study with the variation of temperature indicates a change in the form of hysteresis loops. This is due to the transition from ferromagnetic to superparamagnetic behavior. We found that cubic shaped Co NPs while decorating RGO show the highest values for some critical magnetic parameters. Coercivity, magnetic moment, and squareness ratio are these parameters. Besides, the nanocomposite impregnated aqueous sols are found to be quite stable and could be a potential candidate for inkjet printing and ferrofluid as the squareness ratio $\left(\mathrm{M}_{\mathrm{r}} / \mathrm{M}_{\mathrm{s}}\right)$ is very small.
\end{abstract}

KEYWORDS: Co-RGO nanocomposites, Co-precipitation, Hydrothermal, Magnetic study, Squareness ratio $(\mathrm{Mr} / \mathrm{Ms})$. 


\section{INTRODUCTION:}

Recently, RGO based metal nanocomposites have gained considerable attention due to their possible scope of applications in particular sensing [1], energy storage [2,3], electro - and photocatalysis [4-6]. Noble metals are most suitable for Graphene/RGO based metal nanocomposites as they delineate high chemical stability, catalytic activity, sensing, etc. However, due to scarcity and high-cost noble metals cannot be the proper choice for large scale production.

Instead, researchers are trying to replace noble metal with $3 \mathrm{~d}$ transition metals as they are readily available and cheap. Amongst others, researchers extensively used cobalt, one of the readily available essential $3 \mathrm{~d}$ metals in the catalysis process. Co nanocomposites is also an excellent candidate for ferrofluid [7], drug delivery [8], hyperthermia treatment [9], and magnetic imaging applications [10].

For such a wide variety of applications, a well-dispersed suspension of magnetic material in a solvent is desirable. For specific applications, the suspension needs to be in the water.

As one s-electron from Co gets promoted to p-level due to its placement in a single defect of graphene, leading to "spd" hybridization [11], which results in the in-plane bonding of three neighboring carbon atoms via d-p mixing between unpaired $6 \mathrm{~d}$ electron from Co with $\mathrm{p}_{z}$ orbital of one of three adjacent carbon atoms. This leads to protection against oxidation of Co when it decorates RGO defects [12]. Besides, this leads to a situation of donor-acceptor configuration in this metal-RGO system. The magnetic nano-materials under such a circumstance do face a problem of clustering, and to avoid this problem, different research groups used various strategies such as surface functionalization, capping, embedding, etc. [13]. One such simplified approach is to synthesize metal/metal oxide decorated reduced graphene oxide (RGO). 
Decoration of metal nanoparticles on the graphene sheets prevents their oxidation \& agglomeration.

Since we are trying to synthesize a relatively stable fluid, impregnated with Co-RGO nanocomposite particles, it is vital to measure their magnetic property, as the stability of the liquid is dependent not only on the structural configuration but also on magnetic properties. In particular, the value of magnetic squareness for good dispersion should be less than 0.5 , whereas, for the recording medium, it needs to be close to 0.5 . Structurally, it is interesting to note that although magnetic NPs and RGO have collapsing tendency, a composite of the two can balance this tendency [14].

We have not used any surfactant or capping agent. This keeps the composite active in terms of applications. A capped configuration would have rendered the suspension far less active, as all the active sites would have been covered with capping agents or surfactants. Controlling the composition of the composites and their magnetic squareness, but without compromising their active sites, we have synthesized a magnetic nanofluid that is stable for a longer time. We report the results of this work in this paper.

\section{EXPERIMENT}

Reagents and materials: We used graphite powder (fine-grained), hydrogen peroxide $\left(\mathrm{H}_{2} \mathrm{O}_{2}\right)$, potassium permanganate $\left(\mathrm{KMnO}_{4}\right)$, sulfuric acid $\left(\mathrm{H}_{2} \mathrm{SO}_{4}\right)$, sodium nitrate $\left(\mathrm{NaNO}_{3}\right)$, and hydrochloric acid for the synthesis of Graphene Oxide (GO). The additional chemicals used are sodium borohydride $\left(\mathrm{NaBH}_{4}\right)$, sodium hydroxide $(\mathrm{NaOH})$, and cobalt chloride hexahydrate $\left(\mathrm{CoCl}_{2} \cdot 6 \mathrm{H}_{2} \mathrm{O}\right)$. We mixed cobalt chloride hexahydrate $\left(\mathrm{CoCl}_{2} \cdot 6 \mathrm{H}_{2} \mathrm{O}\right)$ with the $\mathrm{GO}$ powder. We maintained a high $\mathrm{pH}$ with the addition of $\mathrm{NaOH}$ and executed the reduction with $\mathrm{NaBH}_{4}$. All 
the analytical grade reagents were procured from Sigma-Aldrich and used as received without any further purification.

Synthesis of GO: We synthesized GO by a chemical route named the modified Hummer's method $[15,16]$ with graphite powder as a precursor and sodium borohydride as a reducing agent. Synthesis of RGO-Co composites:

RGO-Co nanoparticles have been synthesized by a simple chemical method using cobalt chloride hexahydrate \& GO powder as precursors and di-ionized (DI) water as a solvent to grow different shapes of cobalt nanoparticles on RGO sheets. Solutions of $0.05 \mathrm{M}$ cobalt chloride hexahydrate and 0.78 weight $\%$ of GO powder were prepared in DI water and stirred to make individual homogenous solutions. Cobalt solution was added into the GO solution and allowed to mix well by stirring for an hour. Subsequently, we added $\mathrm{NaOH}$ into the mixture of precursors, followed by $1 \mathrm{M} \mathrm{NaBH}_{4}$ at constant stirring for half an hour. The mixture was divided into three parts to provide three different treatments to grow particles of different shapes.

\section{a) Spherical Cobalt nanoparticles-RGO composites}

We prepared the spherical nanoparticles at a high $\mathrm{pH}$-based reduction of Co ions in a solution, impregnated with a suitable amount of GO, under room temperature and atmospheric pressure with $\mathrm{NaBH}_{4} . \mathrm{NaBH}_{4}$ reduced both $\mathrm{GO}$ and $\mathrm{Co}^{2+}$ ions simultaneously to $\mathrm{RGO}$ and Co metal. The Co metal NPs decorated the RGO at its edge as well as its basal plane through various functional groups and defects. At equilibrium condition, due to surface energy minimization, the Co NPs

became spherical. The size control was due to the simultaneous conversion and decoration of various defects and functional groups. If it was a simple reduction of Co salts, it could have created varied size, whereas in this case, as there was already nucleation sites on GO, a stricter 
size distribution resulted $[17,18]$. We washed the precipitate several times using DI water at $90^{\circ} \mathrm{C}$, and the resulting black powder was collected [19].

\section{b) Cobalt nanorod-RGO composites}

To synthesize nanorod like structures, with the same experimental conditions as mentioned in spherical cobalt nanoparticles-RGO composites, we additionally applied a 0.6 Tesla magnetic field for 60 minutes. The $\mathrm{GO}$ suspended solution $\mathrm{Co}^{2+}$ ions at room temperature and pressure, at a high $\mathrm{pH}$, was subjected to this magnetic field by putting the reaction mixture within two magnetic poles. We reduced the initial solution with $\mathrm{NaBH}_{4}$. The sample was washed and dried at $90^{\circ} \mathrm{C}$ in the oven for 24 hours, and the black powder was collected.

Initially, below a single particle domain size, at a small size regime, the particles were superparamagnetic. To minimize the magneto-static energy, they develop a tendency to grow in the direction of the magneto-crystalline easy axis, which tended to align following field lines within the solution. If the final size were such that the particles became ferromagnetic, then the aligned structures would remain partially intact in the composites. Otherwise, with the magnetic field withdrawn, they would randomize and rearrange within the solution, provided they were superparamagnetic. However, all the samples have contributions from ferromagnetic and superparamagnetic order. Therefore, the structure remained partially intact $[20,21]$.

\section{c) Cobalt nanocube-RGO composites:}

Typically, we need to avoid equilibrium thermodynamic conditions to create anisotropic shapes of Co NPs. We took a different pathway for synthesizing the cubic Co NPs on RGO. By using the hydrothermal route, we reached the non-equilibrium condition. Instead of decomposing Cobased carbonyl compounds, we took a bottom-up approach where $\mathrm{Co}^{2+}$ ions in the solution from 
$\mathrm{CoCl}_{2}$ salts were directly reduced by $\mathrm{NaBH}_{4}$ simultaneously with $\mathrm{GO}$ under non-equilibrium hydrothermal condition. The particles were prepared by transferring this mixture into Teflonlined stainless steel autoclave and then kept the autoclave into an oven at $90^{\circ} \mathrm{C}$ temperatures for 24 hrs. The sample was cooled down and centrifuged to wash the byproducts. We dried the sample was dried in the oven at $90^{\circ} \mathrm{C}$, and the resulting black sample powder was collected.

Here, taking a cue from Fischer-Tropsch synthesis procedure for cubic shaped Co NPs on $\mathrm{TiO}_{2}$ support [22], we had grown Co cubic nanostructures on GO support, which at the end got converted to RGO due to reduction.

In hydrothermal synthesis, cube-shaped $\mathrm{Co}_{3} \mathrm{O}_{4}$ forms with high $\mathrm{pH}$ without reducing agent. Under optimized conditions for hydrothermal synthesis, in the presence of a reducing agent such as $\mathrm{NaBH}_{4}$, a two-stage reduction to cubic shaped Co NPs happened. The phase of Co NPs is hexagonal, found by XRD analysis. Under high $\mathrm{pH}$ conditions, Go was reduced to RGO simultaneously. The RGO basal planes and edges were decorated by Co nanocubes, as observed in other cases. The schematic in Figure 1 provides a snapshot of all the processes followed for synthesis and mechanism of growth of various shapes of Co NPs in the Co NPs-RGO composites, dispersed in water. 


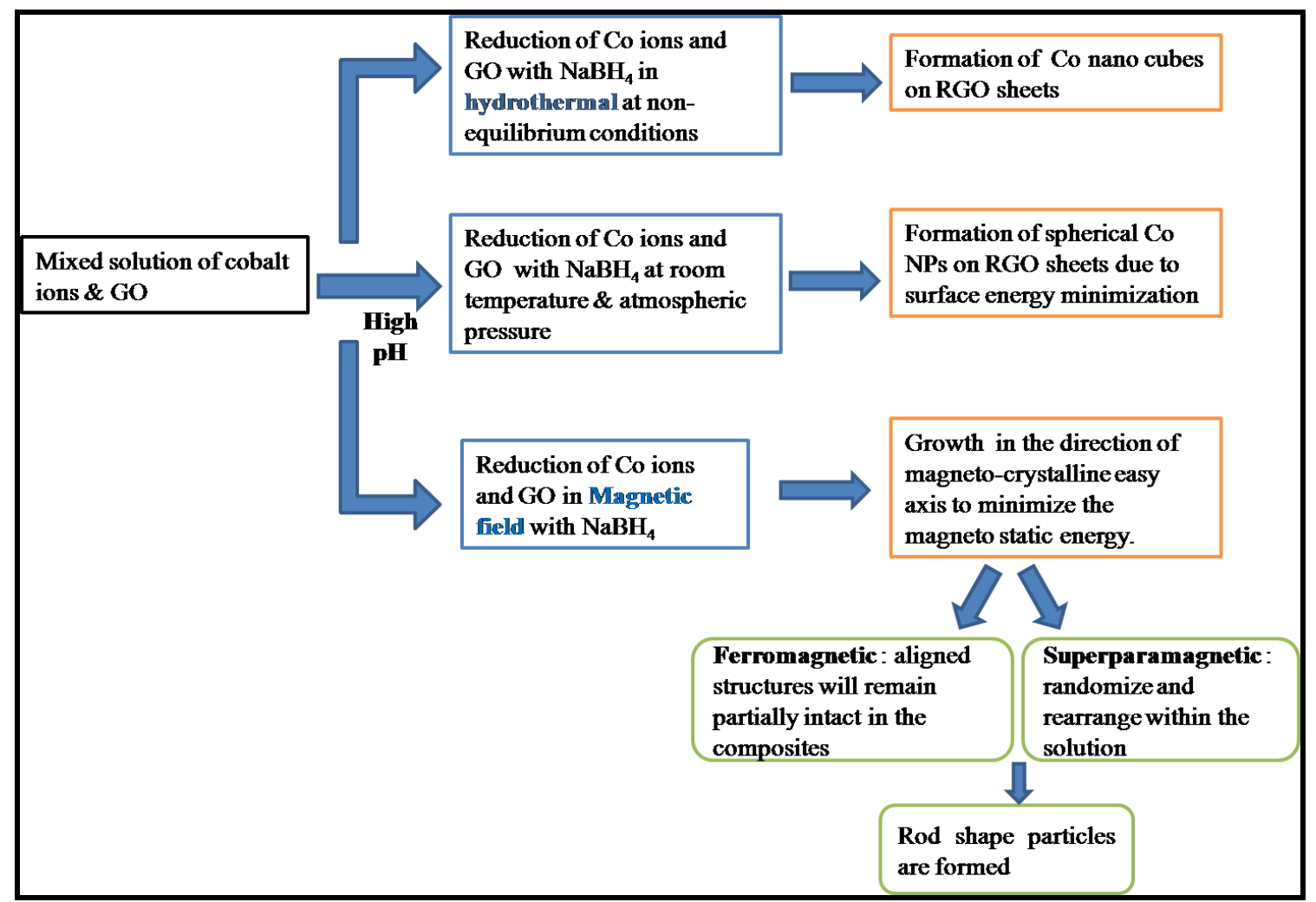

Figure 1: Schematic diagram of the synthesis of RGO-Co NPs by different methods

Characterization Techniques: We characterized the synthesized RGO-Co composites by different sophisticated techniques. We used the X-ray diffraction technique to determine the phase formation and purity of the prepared nanocomposites. We used X Pert Pro X-ray diffractometer; (PANALYTICAL, Almelo, The Netherlands) fitted with $\mathrm{Cu} \operatorname{K\alpha }(\lambda=1.54 \AA$ A $)$ target and nickel filter. We performed FTIR spectroscopy at room temperature to detect the vibrational spectra of all the prepared samples using Nicolet Magma-IR (750, Series II) spectrometer in the range $400-4000 \mathrm{~cm}^{-1}$. Raman spectrometer (Invia Renishaw) captured the Raman spectra of the samples using a laser source of $\mathrm{He} / \mathrm{Cd}$ of $514.5 \mathrm{~nm}$ excitation.

We performed the transmission electron microscope (TEM) measurements using TEM-JEOLEM-2100 (operating voltage of $200 \mathrm{kV}$ ) for the morphological and structural analysis of the 
samples. We cast a drop of nanocomposites on carbon-coated TEM grids procured from TedPella for TEM study. As the samples are drop cast, the arrangement of Co-RGO nanocomposite particles become random while drying. We measured the magnetic properties of these nanocomposites by Quantum Design SQUID MPMS VSM. Magnetization versus temperature measurement has been recorded both in field cooled (FC) and zero-field cooled (ZFC) conditions.

\section{RESULTS \& DISCUSSIONS}

Composites of cobalt nanoparticles embedded in reduced graphene oxide sheets were synthesized by three different methods, namely co-precipitation method at room temperature, by applying the magnetic field to grow cobalt nanoparticles while co-precipitating, and the hydrothermal method at $90^{\circ} \mathrm{C}$. In this paper, we have studied the effect of the synthesis method and magnetic properties of cobalt nanoparticles anchored on RGO surfaces. The three samples used are respectively, spherical Co-RGO, nanorod Co-RGO, and cube-shaped Co-RGO nanocomposites.

\section{X-Ray Diffraction Study (XRD):}

For structural characterization of Co-RGO nanocomposites, we performed XRD scan in the range of $20-70^{\circ}$ and shown in figure 2 . We have also performed an XRD in the same range for RGO for comparison as base material. While synthesizing the RGO by reducing GO, the characteristic peak of GO disappears and two broad peaks appear for RGO near $26^{\circ}$ and $42^{\circ}$ corresponds to 002 and 100 lattice planes [6,23]. Chemically reduced Graphene oxide shows the characteristic peak for RGOIGraphene near 26 degree with increased interlayer spacing than graphite corresponds to $\pi-\pi$ restacking [24] and shows stack structure of RGO attributed to $\mathrm{H}$ atom and aromatic $\mathrm{OH}$ bonding [25]. 
The cobalt nanoparticles form the hexagonal system, confirmed by standard data (PCPDF File No. 897373). We observed prominent peaks of Co NPs at $44 \& 47^{\circ}$ for (002) \& (101) planes, respectively [26].

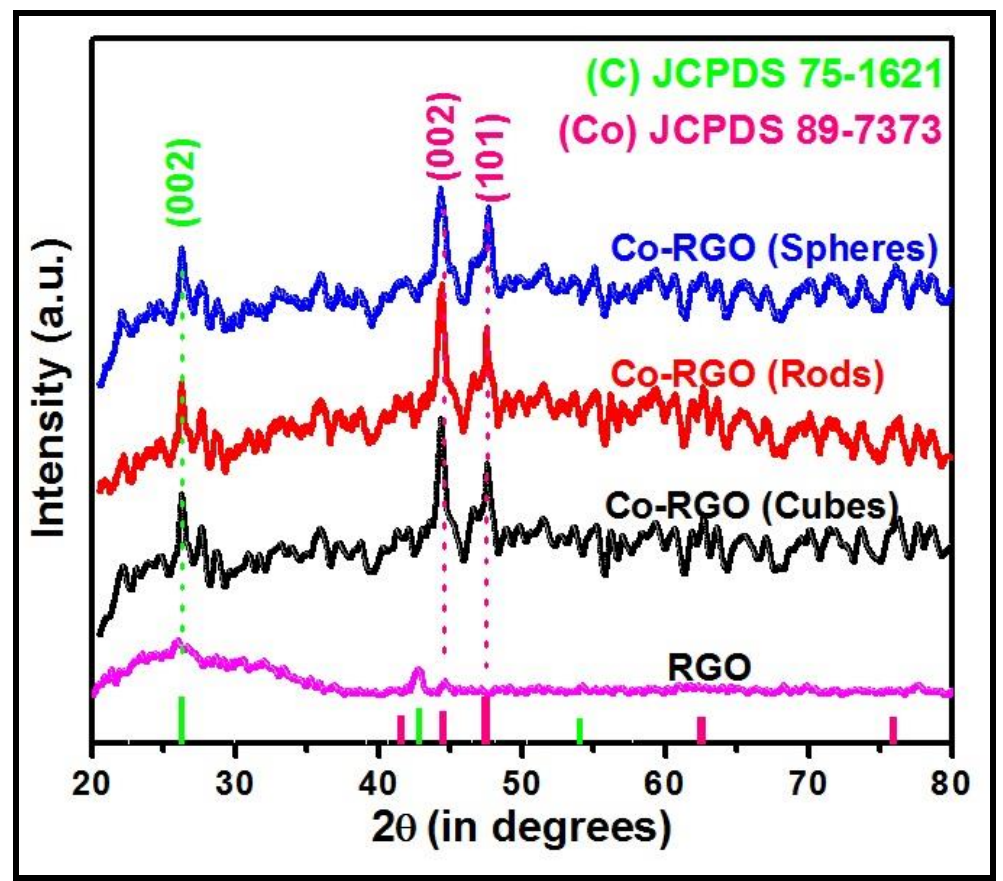

Figure 2: X-ray diffraction patterns of RGO and Co-RGO composites (Spherical Co-RGO, Rod Co-RGO, and Cubic shaped Co-RGO indicated with the graph)

Transmission Electron Microscopy (TEM): We carried out the morphology and structural analysis of graphene-based cobalt composites using TEM/ HRTEM. TEM images of sample RGO-Co are presented in Figure 3, which shows the densely decorated RGO sheet by cobalt nanoparticles. 


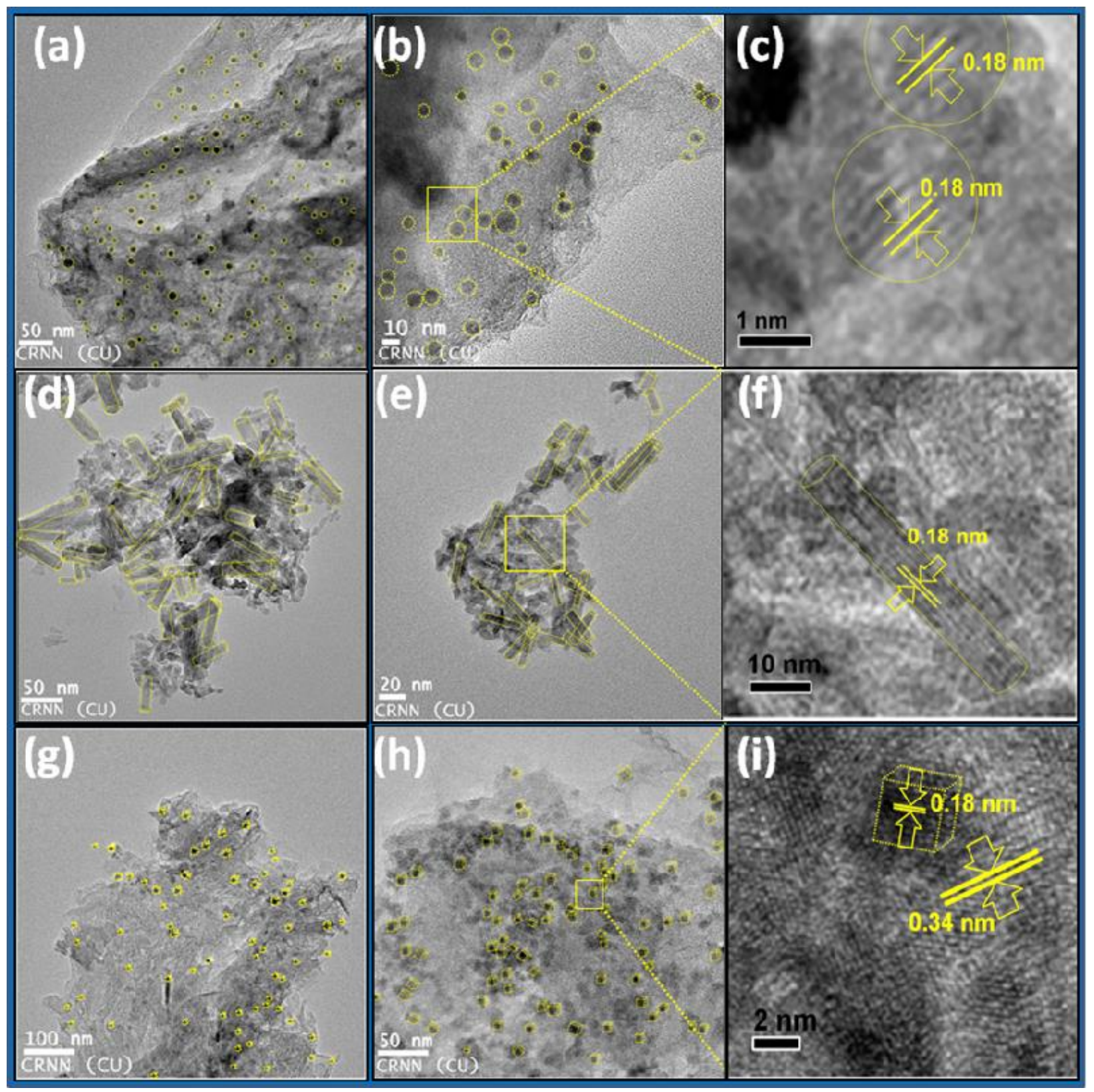

Figure 3. Transmission electron micrograph in various scale, with associated lattice image (Scale as indicated in the image): 3 (a), (b), (c) Spherical Co-RGO synthesized by co-precipitation method; 3 (d), (e), (f) Rod-like Co-RGO synthesized by co-precipitaion with applied magnetic field; 3 (g), (h), (i) Cubic Shaped Co-RGO nanocomposites synthesized by hydrothermal method. The boundaries are for visual guidance. 
Figure 3(a) \& (b) show tiny cobalt nanoparticles of spherical shape with an average particle size of $6 \mathrm{~nm}$ decorating RGO sheets in two different scales, indicated in the figure. Figure 3(c) reveals lattice spacings the Co NPs $(0.18 \mathrm{~nm}$, assigned (002) plane) [27] which corroborates well with the findings of XRD analysis. Figure 3(d), and (e) show the formation rod-like Co NPs on RGO surface, in two different magnification. Figure 3 (f) shows the lattice plane of the rodlke Co NPs, matching with (002) of Co nanocrystals. Figure 3 (g), (h) are representation of cubic shaped Co NPs on On RGO surface, in two different scales, while 3 (i) reveals lattice spacings both the Co NPs (0.18 nm, assigned (002) plane) and RGO ( $0.34 \mathrm{~nm}$ assigned to (111) planes $[28,29]$.

The prepared cubes are also very tiny, and the dimension is below $10 \mathrm{~nm}$ for some of them, while there are some bigger particles too. We found that the estimated average length of Co nanorods is around $123 \mathrm{~nm}$, the width being $\sim 6 \mathrm{~nm}$.

FTIR Spectroscopy: We present the FTIR spectrum in the range $500-4000 \mathrm{~cm}^{-1}$ of GO, RGO, and Co-RGO composites in Figure 4. Characteristics peaks of GO at $\left(1725 \mathrm{~cm}^{-1}\right) \mathrm{C}=\mathrm{O}, \mathrm{C}-\mathrm{O}$ $\left(1065 \mathrm{~cm}^{-1}\right), \mathrm{C}=\mathrm{C} \quad\left(1634 \mathrm{~cm}^{-1}\right)$ correspond to aromatic bond, and those are absent in the FTIR spectrums of all the samples other than for GO, which indicate the reduction of GO to RGO [29]. FTIR peak at $1388 \mathrm{~cm}^{-1}$ corresponds to a deformation of the O-H group for Co-RGO nanocomposites splits and shift to $1421 \mathrm{~cm}^{-1}$ for RGO. A sharp band at $3430 \mathrm{~cm}^{-1}$ corresponds to $\mathrm{O}-\mathrm{H}$ stretching, which is most prominent in GO. This band diminishes for composites as compared to RGO. The presence of a peak at $562 \mathrm{~cm}^{-1} \& 661 \mathrm{~cm}^{-1}$ for all the composites correspond to C-OH out of plane bending. The presence of peaks at $1635 \mathrm{~cm}^{-1}$ for GO shifted to $1578 \mathrm{~cm}^{-1}$ correspond to $\mathrm{C}=\mathrm{C}$ aromatic symmetrical stretching, and the presence of a maximum at $1026 \mathrm{~cm}^{-1}$ corresponds to C-C bands. Sodium borohydride removes the epoxy and hydroxyl 
groups of GO and RGO is produced. Simultaneously this process is responsible for the creation of topological defects in $\mathrm{sp}^{2}$ islands [30].

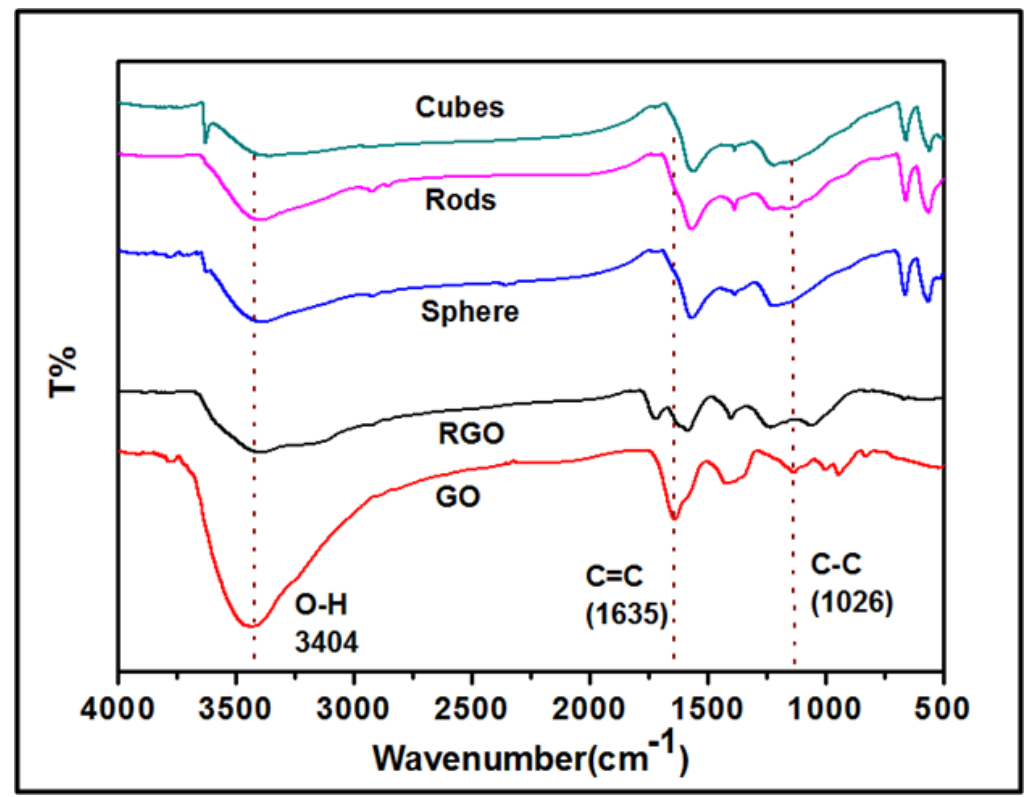

Figure 4: FTIR spectra of GO, RGO, Co-RGO composites synthesized by different methods.

Raman Spectroscopy: Number of layers, level of doping and defects present in the graphenebased systems can be studied with the help of Raman spectrums [31]. The Raman spectrums for GO, RGO \& RGO-Co composites are shown in Figure 5. The characteristics Raman band of graphene is called D band, which appears for all the samples near $1351 \mathrm{~cm}^{-1}$. The D band appears due to defects \& disorder present in the system. The D band is not, however, a characteristics peak of graphitic materials, as it violets the selection rule [32]. The primary feature of Raman spectra for graphene-based samples is the G band, which appears near 1584 $\mathrm{cm}^{-1}$. This $\mathrm{G}$ band arises due to first-order scattering of $\mathrm{E}_{2 \mathrm{~g}}$ vibrational mode and shift to $3-5 \mathrm{~cm}^{-1}$ for single layer Graphene [33]. 
For Co-RGO composites of different shapes, the $\mathrm{G}$ band peaks shift towards higher wavenumber at $1594 \mathrm{~cm}^{-1}$. When GO is reduced to RGO simultaneously with the formation of Co NPs or independently, a new set of C-C vibration may form, which is represented by the shift shown by the $\mathrm{G}$ band. This unique vibration mode was assigned to alternative single double bonds of carbon within $\mathrm{sp}^{2}$ clusters, Stone-Wales and 5-8-5 defect structures [34].

The ratios of intensities of D \& G peaks are 1.012, 0.997, 0.994, and 0.8 for RGO-Co composites of various shapes, namely sphere, cubes, rods, and GO, respectively. The higher value of $\mathrm{ID}_{\mathrm{D}} / \mathrm{IG}_{\mathrm{G}}$ for RGO-Co composites as compared to GO shows the decrease of the $\mathrm{sp}^{2}$ domain of GO after reduction [6] and gives a measure of disorder. The increased value of ID/IG signifies the possible emergence of increasing luminescent defects with a reduction [35,36].

The $2 \mathrm{D}$ band is the second order of phonon mode which appears at $2705 \mathrm{~cm}^{-1}$ for GO in Figure 4(a) but changes into small hump for composites. The presence of a peak at lower wavenumber near $680 \mathrm{~cm}^{-1}$ corresponds to the deformation modes of a metal-oxygen group.

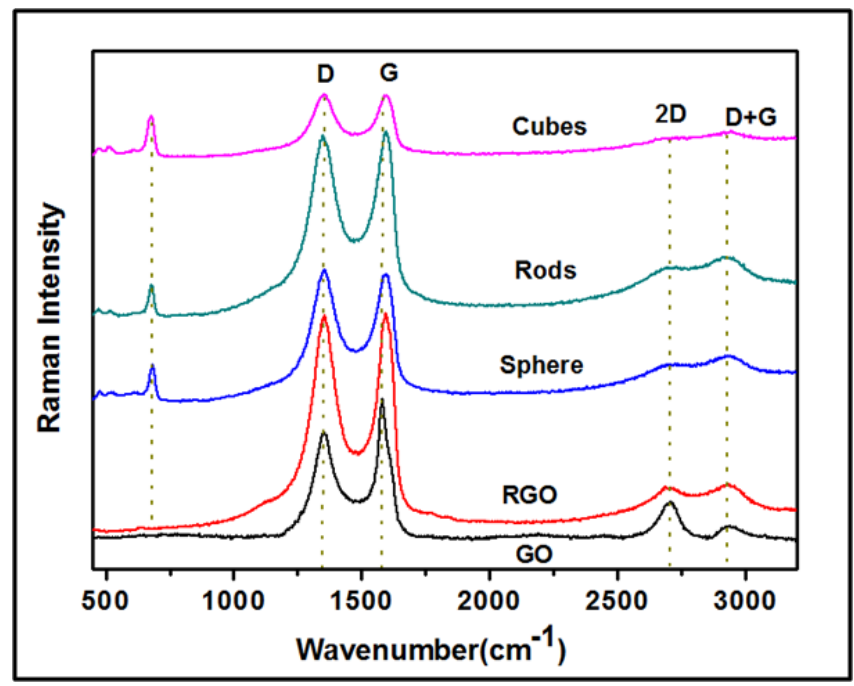

Figure 5: Raman spectra of GO, RGO, and RGO-Co composite synthesized by different methods 


\section{Magnetic Characterization:}

We carried out the magnetic study of all the prepared samples in the temperature range from $5 \mathrm{~K}$

to $300 \mathrm{~K}$, as shown in Figure 6. All the hysteresis loops exhibit the characteristic features of ferromagnetic materials. We observed a distinct variation in magnetic interaction from the change of hysteresis loop with temperature. A ferromagnetic type hysteresis loop is noted for all the nanocomposites while measured at $5 \mathrm{~K}$. However, the samples change from ferromagnetic to superparamagnetic with an increase of temperature. All the $\mathrm{M}-\mathrm{H}$ curves, except for $5 \mathrm{~K}$, appear like a straight line. The $\mathrm{M}-\mathrm{H}$ curves have almost negligible coercivity, squareness ratio, and remanence. Such behavior indicates the superparamagnetic nature.
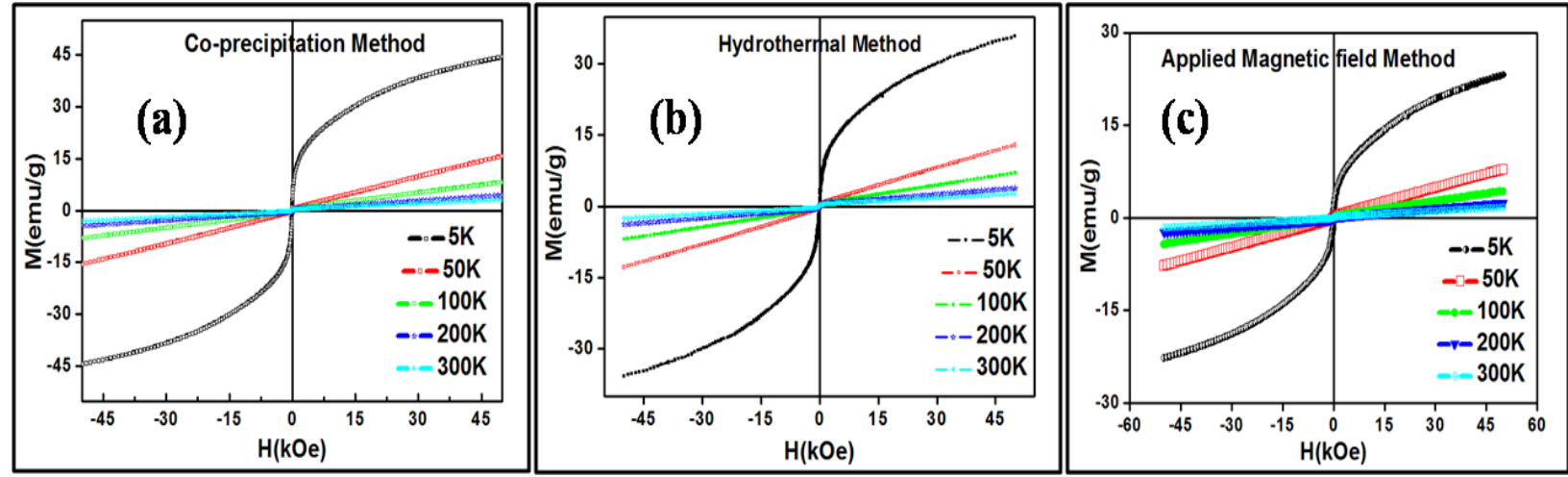

Figure 6: Magnetic hysteresis loop at 5K, $50 \mathrm{~K}, 100 \mathrm{~K}, 200 \mathrm{~K} \& 300 \mathrm{~K}$ for RGO-Co sample prepare by (a) Co-precipitation method (b) Hydrothermal method (c) Applied magnetic field with co-precipitation.

The measured hysteresis loop at $5 \mathrm{~K}$ of all the samples is also not conventional, as observed for Ferri/Ferromagnetic materials. Magnetization does not saturate even at an applied magnetic field of 5 Tesla. This behavior indicates the presence of both ferromagnetic (FM) as well as superparamagnetic (SPM) phases [37,38]. To separate the superparamagnetic (SPM) part from ferromagnetic (FM) contribution, we employed the following relation: 
$M(H)=\left[2 \frac{M_{F M}^{S}}{\pi} \tan ^{-1}\left\{\left(\frac{H \pm H_{C i}}{H_{c i}}\right) \tan \left(\frac{\pi M_{F M}^{R}}{2 M_{F M}^{S}}\right)\right\}\right]+\chi \mathrm{H}$

Where $M_{F M}^{S_{F M}}$ is the FM saturation magnetization, $M_{F M}^{R}$ is remanence magnetization, $H_{c i}$ is coercivity, and $\chi$ is the susceptibility of the SPM part.

We depicted the fitted and simulated curves in Figure 7. The presence of a significant fraction of SPM for all the samples is inhibiting to get saturation in M-H curves.
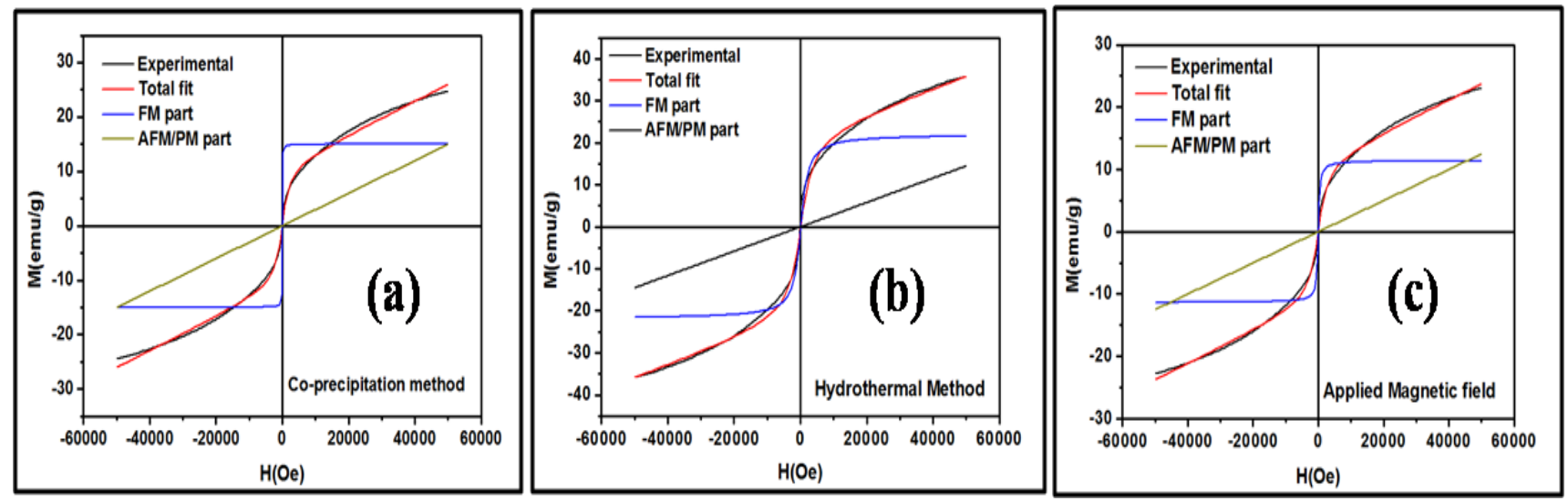

Figure 7: The FM and SPM simulated curves along with their fitting for samples prepared by (a) Co-precipitation method (b) Hydrothermal method (c) Applied Magnetic field with coprecipitation measured at $5 \mathrm{~K}$

We point out that the change in morphology also affects the anisotropy. The anisotropy, in turn, reflects in the coercivity of the nanoparticles. We used simulated FM parts to extract the anisotropy information. We then applied the law of approach to the saturation magnetization for this purpose [39]:

$M=M_{s}\left(1-\frac{A}{H}-\frac{B}{H^{2}}\right)+\kappa H$

Where $M$ is the magnetization near the saturation magnetization, $H$ is the applied magnetic field, and $M_{S}$ is the saturation magnetization, $\mu_{0}$ is the permeability of the free space. 
The first term $A / H$ is valid only for a finite range of applied field strength as inhomogeneity, crystal defects, etc., if present in the system, will be diminished as the applied field gets sufficiently higher. The last term is applicable at higher temperatures and higher fields. This term comes into effect due to the impurities contained in the material. The moments associated with the contaminants aligned themselves by force along the direction of the applied field at higher values. We, therefore, used Eqn. 2 by considering $B / H^{2}$ term. For uniaxial anisotropy $B=\frac{4 K^{2}}{15 M_{s}{ }^{2}}$

The results for different samples are tabulated in Table 1.

Table 1: Various magnetic properties for Co-RGO nanocomposites prepared by various methods

\begin{tabular}{llll}
\hline $\begin{array}{l}\text { Various } \\
\text { important } \\
\text { parameters }\end{array}$ & Co-precipitation & $\begin{array}{l}\text { Applied magnetic } \\
\text { field with co- } \\
\text { precipitation }\end{array}$ & Hydrothermal \\
\hline Ms & 14.99 & 11.33 & 21.54 \\
\hline Hc & 35 & 51 & 151 \\
\hline $\mathbf{M r}$ & 23.54 & 21.22 & 45.94 \\
\hline $\begin{array}{l}\text { K1 } \\
\text { (anisotropy } \\
\text { energy) erg/cc }\end{array}$ & 24954 & 53554 & 264771 \\
\hline
\end{tabular}

We investigated the temperature dependence of the magnetization process through FC-ZFC measurements of the samples. We used a magnetic field of magnitude $0.1 \mathrm{kOe}$, in the temperature range $5 \mathrm{~K}$ to $300 \mathrm{~K}$. As a result, we could study the superparamagnetic behavior of these samples in detail. For the ZFC study, we cooled the samples first down to $5 \mathrm{~K}$ in the absence of any applied field, and then we measured the magnetic response while heating slowly 
in the presence of an applied magnetic field of $0.1 \mathrm{kOe}$. On the other hand, we carried out field cooled study after cooling the samples in the same applied field of which was used for measurement $(0.1 \mathrm{kOe})$ while heating. All the nanocomposites delineate similar characteristic behavior of superparamagnetic particles, shown in Figure 8. The ZFC curves show a clear maximum at a particular temperature called a blocking temperature of the material. This result indicates a transition to the unblocked state from a blocked state implies Ferro /Ferri to SPM states.

In contrast, the FC curve continues to increase below blocking temperature, which indicates the absence of spin-glass states. This phenomenon occurs due to the sufficient thermal energy which overcomes field particle coupling. The blocking temperatures for the samples synthesized through hydrothermal, co-precipitation, and by applying a magnetic field are very close to each other and found to be $6.7,7.11$, and $6.4 \mathrm{~K}$, respectively. This result corroborates with the fielddependent magnetic study, which delineates ferromagnetic behavior only at $5 \mathrm{~K}$ for all the samples.
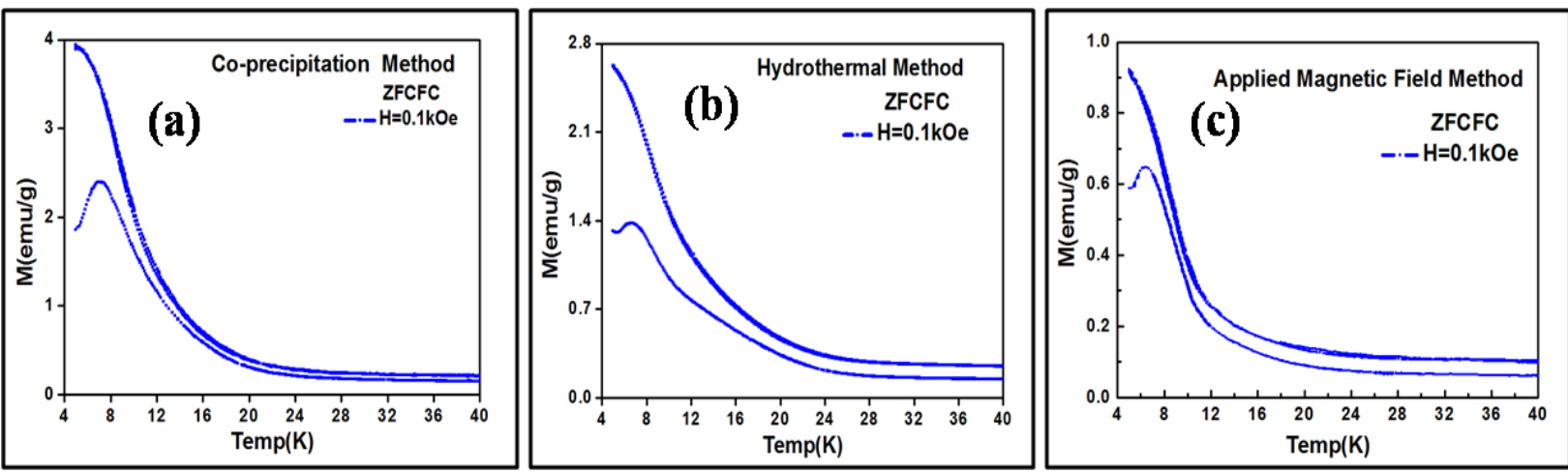

Figure 8: FC-ZFC curve at applied field $0.1 \mathrm{kOe}$ for RGO-Co sample prepare by (a) Coprecipitation method, (b) Hydrothermal method (c) Applied magnetic field with co-precipitation. 
We obtained the switching field distribution from subtracted FM part of the hysteresis by plotting $\mathrm{dM} / \mathrm{dH}$ vs. $\mathrm{H}$ and delineated in Figure 9. All the samples showed a narrow distribution of a single peak near zero. The most substantial peak appeared for the samples prepared by the hydrothermal method, which implies the existence of an intense exchange interaction inside this sample [40], shown in Figure 9.

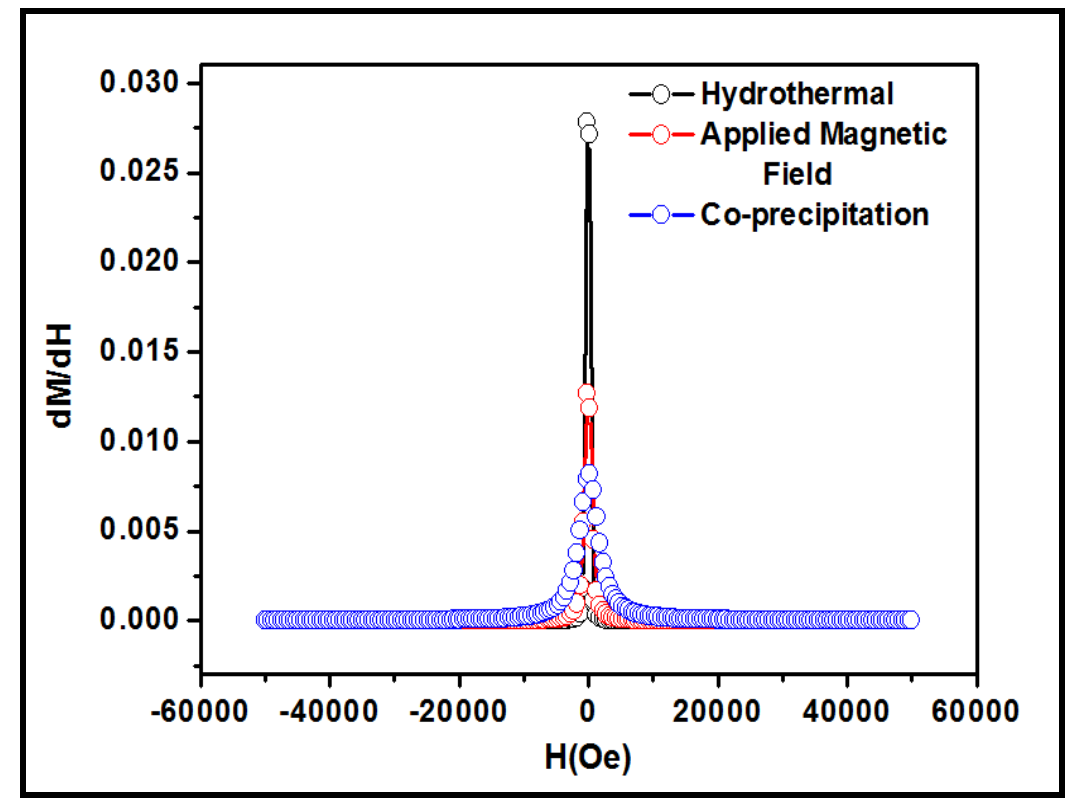

Figure 9: The $\mathrm{dM} / \mathrm{dH}$ versus $\mathrm{H}$ curves for all the nanocomposites

Squareness ratio $(\mathrm{SQR})$ or remnant ratio $(\mathrm{Mr} / \mathrm{Ms})$ of a material is essentially a measure of squareness of the hysteresis loop, which represents the random arrangement of uniaxial particles along with the cubic magneto-crystalline anisotropy. In general, the recording medium needs large SQR values, and the ferrofluid needs small SQR values. In the present work, the squareness ratios of these nanocomposites were very low and varied from 0.016 to 0.053 for different morphologies. A very low value of SQR would be promising for inkjet printing and ferrofluid application. 
Detail magnetic analysis for all the samples delineates that the sample prepared through the hydrothermal process (cubic shape) has superior magnetic properties in terms of Ms, Hc as well as K1. We put in the effort to check the stability of the aqueous solution of these nanocomposites. We displayed a photograph of these aqueous solutions starting from as prepared up to four months is presented in Figure 10. All these solutions remain quite stable up to around one month. After one month, some settlement is observable, which becomes pronounced after four months. However, this settlement is not due to any agglomeration. An ultra-sonication can restore all the suspensions, which once again remain stable.
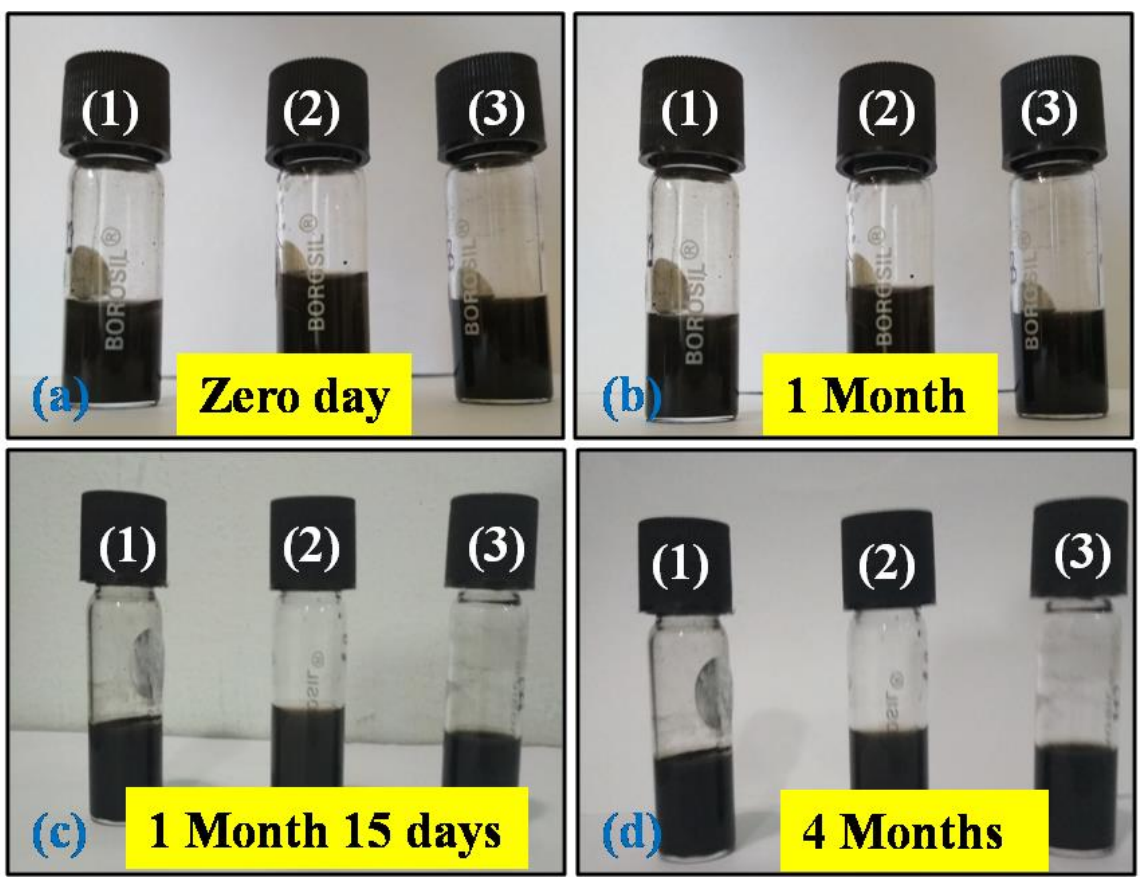

Figure 10: Dispersed solution of RGO-Co composites prepared by three different methods (Coprecipitation method, hydrothermal method and applied magnetic field method mention as 1,2 and3respectively) (a) dispersed solution of samples after one month (b) after one month and fifteen days (c) after four months with the application of ultra-sonication. 
4. CONCLUSION: Reduced Graphene oxide sheets decorated with cobalt nanoparticles of different controlled shapes and sizes have been synthesized successfully by simple chemical methods. TEM image of RGO-Co composites confirmed the embedded decoration of different shapes of cobalt nanoparticles like sphere, cube, and rods on RGO sheets. We have studied the Magnetic properties of RGO-Co composites by varying both the magnetic field and temperature. The pattern of hysteresis loops obtained from the M-H curve indicates ferromagnetic behavior at low temperatures. All the samples show superparamagnetic behavior at high temperatures. FCZFC study also supports this behavior with a blocking temperature below $10 \mathrm{~K}$ for all the samples. The observed squareness ratio for all the samples was minimal and varied from 0.016 to 0.053. We were able to make quite stable dispersion of the nanocomposites in an aqueous medium, which could be very promising for ferrofluid based application.

CONFLICT OF INTEREST: The authors declare that there is no conflict of interest involved in this work.

ACKNOWLEDGMENT: Neelam Singh is thankful to Guru Gobind Singh Indraprastha University, New Delhi for providing financial assistance in the form of Indraprastha Research Fellowship (IPRF). Prof Datta is grateful to the Guru Gobind Singh Indraprastha University, New Delhi for the FRGS grant (GGSIPU/DRC/FRGS/2019/8) and the grant of DST for FIST grant (SR/FST/PSI-167/2011(C). Authors are thankful to the Center for Research in Nanoscience and Technology, University of Calcutta, India, for helping in TEM measurements. 


\section{REFERENCES}

1. H. B. Jeon, P. V. Tsalu, and J. W. Ha, Sci. Rep. 9, 13635 (2019).

2. Y. Li, B. Tan, and Y. Wu, Nano Lett. 8, 265 (2008).

3. M. M. Shahid, P. Rameshkumar, A. Pandikumar, H. N. Lim, Y. H. Ng, and N. M. Huang, J. Mater. Chem. A 3, 14458 (2015).

4. A. Pendashteh, M. F. Mousavi, and M. S. Rahmanifar, Electrochimica Acta 88, 347 (2013).

5. J. L. S. Gascho, S. F. Costa, A. A. C. Recco, and S. H. Pezzin, J. Nanomater. 2019, 1 (2019). 6. M. M. Shahid, A. Pandikumar, A. M. Golsheikh, N. M. Huang, and H. N. Lim, RSC Adv 4, 62793 (2014).

7. I. Torres-Díaz and C. Rinaldi, Soft Matter 10, 8584 (2014).

8. N. Venkatesha, P. Poojar, S. Geethanath, and C. Srivastava, Mater. Res. Express 1, 045008 (2014).

9. M. Hoehn, E. Kustermann, J. Blunk, D. Wiedermann, T. Trapp, S. Wecker, M. Focking, H. Arnold, J. Hescheler, B. K. Fleischmann, W. Schwindt, and C. Buhrle, Proc. Natl. Acad. Sci. 99, 16267 (2002).

10. G. Wang, Y. Ma, Z. Wei, and M. Qi, Chem. Eng. J. 289, 150 (2016).

11. A. V. Krasheninnikov, P. O. Lehtinen, A. S. Foster, P. Pyykkö, and R. M. Nieminen, Phys. Rev. Lett. 102, (2009).

12. A. J. Akhtar, A. Gupta, B. Kumar Shaw, and S. K. Saha, Appl. Phys. Lett. 103, 242902 (2013).

13. Y. Yao, C. Xu, J. Qin, F. Wei, M. Rao, and S. Wang, Ind. Eng. Chem. Res. 52, 17341 (2013).

14. J. Ding, B. Li, Y. Liu, X. Yan, S. Zeng, X. Zhang, L. Hou, Q. Cai, and J. Zhang, J. Mater. Chem. A 3, 832 (2015).

15. W. S. Hummers and R. E. Offeman, J. Am. Chem. Soc. 80, 1339 (1958).

16. S. Abdolhosseinzadeh, H. Asgharzadeh, and H. Seop Kim, Sci. Rep. 5, (2015).

17. L. T. Lu, L. D. Tung, I. Robinson, D. Ung, B. Tan, J. Long, A. I. Cooper, D. G. Fernig, and

N. T. K. Thanh, J. Mater. Chem. 18, 2453 (2008).

18. N. Shukla, E. B. Svedberg, J. Ell, and A. J. Roy, Mater. Lett. 60, 1950 (2006).

19. O. Mondal, S. Mitra, A. Datta, D. Chakravorty, and M. Pal, Mater. Des. 101, 204 (2016).

20. C. Jiang, C. W. Leung, and P. W. T. Pong, Nanoscale Res. Lett. 11, (2016).

21. S. L. Tripp, S. V. Pusztay, A. E. Ribbe, and A. Wei, J. Am. Chem. Soc. 124, 7914 (2002).

22. J. A. Delgado, C. Claver, S. Castillón, D. Curulla-Ferré, V. V. Ordomsky, and C. Godard, Appl. Catal. Gen. 513, 39 (2016).

23. Z.-S. Wu, W. Ren, L. Wen, L. Gao, J. Zhao, Z. Chen, G. Zhou, F. Li, and H.-M. Cheng, ACS Nano 4, 3187 (2010).

24. J. L. S. Gascho, S. F. Costa, A. A. C. Recco, and S. H. Pezzin, J. Nanomater. 2019, 1 (2019). 25. P.-G. Ren, D.-X. Yan, X. Ji, T. Chen, and Z.-M. Li, Nanotechnology 22, 055705 (2011).

26. H.-H. Huang, R. K. Joshi, K. K. H. De Silva, R. Badam, and M. Yoshimura, J. Membr. Sci. 572, 12 (2019).

27. D. Matsuura and T. Kizuka, J. Nanomater. 2012, 1 (2012).

28. H.-H. Huang, R. K. Joshi, K. K. H. De Silva, R. Badam, and M. Yoshimura, J. Membr. Sci. 572, 12 (2019).

29. O. Mondal, S. Mitra, M. Pal, A. Datta, S. Dhara, and D. Chakravorty, Mater. Chem. Phys. 161, 123 (2015). 
30. C. Gómez-Navarro, J. C. Meyer, R. S. Sundaram, A. Chuvilin, S. Kurasch, M. Burghard, K. Kern, and U. Kaiser, Nano Lett. 10, 1144 (2010).

31. N. Singh, D. Kothari, J. R. Ansari, M. Pal, S. Mandal, S. Dhara, and A. Datta, J. Phys. Chem. C 123, 10557 (2019).

32. O. Mondal, S. Mitra, M. Pal, A. Datta, S. Dhara, and D. Chakravorty, Mater. Chem. Phys. 161, 123 (2015).

33. M. J. Allen, V. C. Tung, and R. B. Kaner, Chem. Rev. 110, 132 (2010).

34. K. N. Kudin, B. Ozbas, H. C. Schniepp, R. K. Prud'homme, I. A. Aksay, and R. Car, Nano Lett. 8, 36 (2008).

35. Y. Zhou, Q. Bao, L. A. L. Tang, Y. Zhong, and K. P. Loh, Chem. Mater. 21, 2950 (2009). 36. T. Van Khai, H. G. Na, D. S. Kwak, Y. J. Kwon, H. Ham, K. B. Shim, and H. W. Kim, J. Mater. Chem. 22, 17992 (2012).

37. K. L. Routray and D. Behera, J. Mater. Sci. Mater. Electron. 29, 14248 (2018).

38. S. R. Mohapatra, A. Swain, C. S. Yadav, S. D. Kaushik, and A. K. Singh, RSC Adv. 6, 112282 (2016).

39. P. Mondal, C. Balomajumder, and B. Mohanty, J. Hazard. Mater. 144, 420 (2007).

40. O. Mondal, S. Mitra, A. Datta, D. Chakravorty, and M. Pal, Mater. Des. 101, 204 (2016). 